\title{
Neurotoxicology: an update on epidemiology, mechanisms, and pathology
}

\author{
Christoph van Thriel $^{1}$ (D)
}

Received: 26 July 2019 / Accepted: 27 July 2019 / Published online: 1 August 2019

c) Springer-Verlag GmbH Germany, part of Springer Nature 2019

The adverse impact of environmental chemicals on the human brain has been recognized as an important endpoint in toxicology. In one of the most influential toxicology textbooks, the authors stated in their introduction that ... "the target organ of toxicity most frequently involved in systemic toxicity is the CNS" [1]. Accordingly, the 2nd edition of "Experimental and clinical neurotoxicology", edited by Peter Spencer and Herbert H. Schaumburg [2], listed more than 450 compounds that are suspected or proven neurotoxins/toxicants in humans. Naturally occurring neurotoxins, such as domoic acid (DA) or tetrodotoxin (TTX), are among the most potent poisons that can be found in nature, whereas organic solvents are among the man-made neurotoxicants shown to be associated with severe damage in the central and peripheral nervous system [3]. Already in the early 1980s, Acta Neuropathologica published human biopsy pictures showing demyelination of giant axons taken from the terminal portion of the musculocutaneous nerve of the leg of a patient chronically exposed to $n$-hexane and methylethylketone [4]. Later, details about specific neuropathological changes after chronic solvent abuse via inhalation could be shown in 88 autopsy cases [5]. In addition to macroscopic findings, such as enlarged ventricles, white matter abnormalities, and cerebral atrophy, the study showed that chronic solvent leukoencephalopathy can be identified by birefringent PAS-staining macrophages and reactive microglia in the white matter. However, the study was not able to disentangle the neuropathological effects of the different solvents that the cases abused simultaneously. Nevertheless, such a neuropathological differential diagnosis was possible in the field of aluminum (Al) neurotoxicity. The neurotoxicity of $\mathrm{Al}$ is well known and described in detail

Christoph van Thriel

thriel@ifado.de

1 Neurotoxicology and Chemosensation, Leibniz Research Centre for Working Environment and Human Factors, TU Dortmund, Ardeystr. 67, 44139 Dortmund, Germany for various endpoints and species [6]. Al exposure has also been linked to the pathogenesis of Alzheimer's Disease (AD) [7]. Aluminum neurotoxicity is also thought to play a role in dialysis-associated encephalopathy (DAE) where Al-containing drugs are used to control hyperphosphatemia, and dialysis dementia has been frequently observed as clinical outcome. Despite the proven neurotoxicity of Al, another well-conducted neuropathology study by Reusche et al. [8] could show that the changes in human brain tissue in DAE patients differed markedly from $\mathrm{AD}$ patients. DAE patients did not show AD-type neurofibrillary tangles (NFT) above the normal or expected age-related changes, even though the Al concentrations in the brain samples were markedly increased. These examples illustrate the valuable contributions of neuropathology to the area of toxicology, in particular neurotoxicology. When searching the electronic archive of Acta Neuropathologica, one can find approximately 120 publications related to neurotoxicology but only a few, like the examples given before, included histopathological analyses in human brain tissue. Moreover, these studies were mostly performed under conditions of high exposures or even intoxication. During the last decades, only a few neurotoxicity studies or reviews have been published that have a strong focus on human neuropathology (e.g., [9]). This deficit has been identified by the former Editor-in-Chief, Werner Paulus, who developed and initiated the idea of a cluster of reviews addressing current hot topics in neurotoxicology. This was a challenging endeavour as there are some differences between these obviously-related disciplines.

\section{Why the disparity between these two disciplines within neuroscience?}

There are some reasons for this weak association between neuropathology and toxicology that are inherent to the scientific and societal aims of toxicology. Toxicology tries to contribute to risk assessment procedures, and consequently, 
studies are needed that determine dose-response relationships between the magnitude of exposure and the probability to cause adverse health effects. While the histopathological examination of other target organs (e.g., liver and lung) is still a cornerstone in toxicological guideline studies (e.g., as proposed by the Organization for Economic Cooperation and Development (OECD) [10]), neurotoxicity testing in rodents also relies on functional or behavioral testing (e.g., motor activity [11]). With respect to animal studies, the various regulatory agencies also provide some guidance regarding neuropathology assessment [12], but these examinations are more often a rough estimate of neuropathological changes not fully exploiting the current state of the art in neuropathology. Accordingly, behavioral tests in animals, which have been suggested as an important, sensitive, and apical endpoint in chemical risk assessment, are used more frequently [13, 14], and "safe" levels of exposure are often derived from these endpoints, as in the case of the solvent toluene [15] that also causes neuropathological effects at higher doses [5]. Recently, and related to the efforts of developing alternative methods in toxicity testing [16], behavioral testing in zebrafish has been proposed as a model to investigate adverse outcomes in a whole organism upon exposure to neurotoxic compounds [17]. Accordingly, neuropathology is often only a minor point in neurotoxicity testing, both in guideline studies and scientific research.

In humans, epidemiological studies of exposed populations (e.g., occupationally or environmentally) are the only source to derive dose-response relationships, and here, the availability of brain tissue is limited. Even in cases like the solvent abusers [5], it is difficult to link individual exposure data to these brain samples. Therefore, in human studies, neurobehavioral testing has been the only or at least most important endpoint for the assessment of adverse health effects to the human brain [18]. In addition to the strong focus on behavioral measures as a "surrogate marker" of impaired brain functions, the deficit of neurophysiological and even pathological human data in neurotoxicology was also caused by (a) the lack of non-invasive methods to investigate brain functions in vivo, and (b) the limited availability of neuroimaging techniques, such as functional and structural Magnetic Resonance Imaging (MRI). Various neurophysiological techniques have become increasingly available in epidemiological studies among workers, e.g., manganese-exposed welders [19], and thereby, the knowledge about neurotoxic mechanisms can be used for the selection of sensitive, neurophysiological endpoints in experimental or epidemiological studies [20, 21]. Later, the validation of such neuroimaging findings in brain tissue could provide conclusive information about dose-dependent neuropathological changes after neurotoxic exposures. Thereby, the impact of neurotoxic exposures could also be evaluated more precisely as these findings can be compared to the neuropathology of aging [22] or neurogenerative diseases [23].

\section{Recent developments that might facilitate interactions between neuropathology and toxicology}

The ongoing paradigm shift towards toxicological testing strategies that are based on mechanistic knowledge about the perturbations of molecular and cellular events within neural cells and networks [24, 25] might promote or revive the integration of neuropathology into toxicology. One conceptional tool that is relevant here is the idea of an "Adverse Outcome Pathway" [26] that has be adopted by neurotoxicity [27]. Here, the molecular initiating event (MIE), e.g., the chronic antagonism of $\mathrm{N}$-methyl-d-aspartate receptors (NMDARs) during brain development, should cause impairment of learning and memory abilities in children. The apical endpoint of this adverse effect might be the reduced IQ of children as shown for low-level lead exposures [28]. Such adverse effects of environmental toxicants have also been addressed in neurophysiological and pathological studies, as summarized for prenatal exposure to maternal cigarette smoking (PEMCS) [29]. In particular, the MRI readouts were able to detect adverse neurotoxic effects in exposed children, such as thinner orbitofrontal, middle frontal, and parahippocampal cortices in smoke-exposed children [30]. These morphometric readouts were more sensitive than the Wechsler Intelligence Scale for Children. Moreover, in this review, neuropathological findings from animal studies were able to provide more details about the pathological changes in the brain of rats (e.g., increased spine density in the granule cells, and terminal and basal dendrites of the pyramidal neurons of CA 3 and CA 1 of the hippocampus). These examples clearly showed that the paradigm change in neurotoxicology might be a chance to intensify the collaboration between neuropathology and neurotoxicology.

The present neurotoxicity review cluster is intended to be a first step in this direction and might encourage researchers from both disciplines to intensify collaborations. Two hot topics from neurotoxicology, namely the exposure to pesticides [19] and polychlorinated biphenyls (PCBs) [31], will be addressed in detail. Both reviews will provide some information about the history of these neurotoxicants, their diversity with respect to chemistry, and their ability to persist in the environment and human tissue even for decades. Summaries of in vivo and in vitro studies describing the different neurotoxic mechanisms that have been discovered are presented, and their relevance for the epidemiological findings has been discussed. While the review on PBC has a stronger focus on developmental neurotoxicity, the pesticide review will also address possible associations of this class 
of neurotoxicants with neurodegenerative diseases. Finally, both reviews focus on the need for a comprehensive characterization of the neurotoxic properties of new chemicals that may be developed to substitute the two groups of chemicals reviewed in this cluster.

I am confident that this cluster of two reviews is an excellent starting point to stimulate the dialogue between these two "neuro" research areas.

\section{References}

1. Klaassen CD (2008) Casarett and Doull's toxicology-the basic science of poisons ( 7 th edn)

2. Spencer PS, Schaumburg HH, Ludolph AC (2000) Experimental and clinical neurotoxicology. 1352

3. White RF, Proctor SP (1997) Solvents and neurotoxicity. Lancet (Lond Engl) 349:1239-1243. https://doi.org/10.1016/S0140 -6736(96)07218-2

4. Vallat JM, Leboutet MJ, Loubet A, Piva C, Dumas M (1981) N-hexane- and methylethylketone-induced polyneuropathy abnormal accumulation of glycogen in unmyelinated axons-report of a case. Acta Neuropathol 55:275-279. https://doi.org/10.1007/ BF00690990

5. Al-Hajri Z, Del Bigio MR (2010) Brain damage in a large cohort of solvent abusers. Acta Neuropathol 119:435-445. https://doi. org/10.1007/s00401-010-0653-6

6. Krewski D, Yokel RA, Nieboer E, Borchelt D, Cohen J, Harry J et al (2007) Human health risk assessment for aluminium, aluminium oxide, and aluminium hydroxide. J Toxicol Env Heal B Crit Rev 10(Suppl 1):1-269. https://doi.org/10.1080/1093740070 1597766

7. Kawahara M, Kato-Negishi M (2011) Link between aluminum and the pathogenesis of Alzheimer's disease: the integration of the aluminum and amyloid cascade hypotheses. Int J Alzheimers Dis 2011:276393. https://doi.org/10.4061/2011/276393

8. Reusche E, Koch V, Lindner B, Harrison AP, Friedrich HJ (2001) Alzheimer morphology is not increased in dialysis-associated encephalopathy and long-term hemodialysis. Acta Neuropathol 101:211-216

9. Perl DP, Olanow CW (2007) The neuropathology of manganeseinduced parkinsonism. J Neuropathol Exp Neurol 66:675-682

10. OECD (2018) Test No. 413: Subchronic inhalation toxicity: 90-day study. OECD

11. OECD (1997) Test No. 424: Neurotoxicity study in rodents. OECD

12. Bolon B, Bradley A, Butt M, Jensen K, Krinke G, Mellon RD (2011) Compilation of international regulatory guidance documents for neuropathology assessment during nonclinical general toxicity and specialized neurotoxicity studies. Toxicol Pathol 39:92-96. https://doi.org/10.1177/0192623310385145

13. Moser VC, MacPhail RC (1990) Comparative sensitivity of neurobehavioral tests for chemical screening. Neurotoxicology 11:335-344

14. Slikker W Jr, Acuff K, Boyes WK, Chelonis J, Crofton KM, Dearlove GE et al (2005) Behavioral test methods workshop. Neurotoxicol Teratol 27:417-427. https://doi.org/10.1016/j.ntt.2005.02.003

15. Seeber A, Schäper M, Zupanic M, Blaszkewicz M, Demes P, Kiesswetter E et al (2004) Toluene exposure below 50 ppm and cognitive function: a follow-up study with four repeated measurements in rotogravure printing plants. Int Arch Occup Environ Health 77:1-9. https://doi.org/10.1007/s00420-003-0452-7

16. Leist M, Hartung T, Nicotera $P$ (2008) The dawning of a new age of toxicology. Altex 25:103-114
17. Bailey J, Oliveri A, Levin ED (2013) Zebrafish model systems for developmental neurobehavioral toxicology. Birth Defects Res Part C Embryo Today Rev 99:14-23

18. Rohlman DS, Lucchini R, Anger WK, Bellinger DC, van Thriel C (2008) Neurobehavioral testing in human risk assessment. Neurotoxicology 29:555-566

19. Criswell SR, Nielsen SS, Warden M, Perlmutter JS, Moerlein SM, Flores HP et al (2017) 18FFDOPA positron emission tomography in manganese-exposed workers. Neurotoxicology. https://doi. org/10.1016/j.neuro.2017.07.004

20. van Thriel C, Quetscher C, Pesch B, Lotz A, Lehnert M, Casjens $S$ et al (2017) Are multitasking abilities impaired in welders exposed to manganese? Translating cognitive neuroscience to neurotoxicology. Arch Toxicol 91:2865-2877. https://doi. org/10.1007/s00204-017-1932-y

21. Yavari F, van Thriel C, Nitsche MA, Kuo MF (2018) Effect of acute exposure to toluene on cortical excitability, neuroplasticity, and motor learning in healthy humans. Arch Toxicol 92:31493162. https://doi.org/10.1007/s00204-018-2277-x

22. Crary JF, Trojanowski JQ, Schneider JA, Abisambra JF, Abner EL, Alafuzoff I et al (2014) Primary age-related tauopathy (PART): a common pathology associated with human aging. Acta Neuropathol 128:755-766. https://doi.org/10.1007/s00401-014-1349-0

23. Jellinger KA (2009) Alzheimer's disease: a challenge for modern neuropathobiology. Acta Neuropathol 118:1-3. https://doi. org/10.1007/s00401-009-0529-9

24. Bal-Price A, Crofton KM, Sachana M, Shafer TJ, Behl M, Forsby A et al (2015) Putative adverse outcome pathways relevant to neurotoxicity. Crit Rev Toxicol 45:83-91. https://doi. org/10.3109/10408444.2014.981331

25. van Thriel C, Westerink RH, Beste C, Bale AS, Lein PJ, Leist M (2012) Translating neurobehavioural endpoints of developmental neurotoxicity tests into in vitro assays and readouts. Neurotoxicology 33:911-924. https://doi.org/10.1016/j.neuro.2011.10.002

26. Ankley GT, Bennett RS, Erickson RJ, Hoff DJ, Hornung MW, Johnson RD et al (2010) Adverse outcome pathways: a conceptual framework to support ecotoxicology research and risk assessment. Environ Toxicol Chem 29:730-741. https://doi.org/10.1002/etc.34

27. Bal-Price A, Meek ME (2017) Adverse outcome pathways: application to enhance mechanistic understanding of neurotoxicity. Pharmacol Ther 179:84-95. https://doi.org/10.1016/J.PHARM THERA.2017.05.006

28. Liu J, Li L, Wang Y, Yan C, Liu X (2013) Impact of low blood lead concentrations on IQ and school performance in Chinese children. PLoS ONE 8:e65230. https://doi.org/10.1371/journ al.pone. 0065230

29. Chang RCC, Ho YS, Wong S, Gentleman SM, Ng HK (2014) Neuropathology of cigarette smoking. Acta Neuropathol 127:5369. https://doi.org/10.1007/s00401-013-1210-x

30. Toro R, Leonard G, Lerner JV, Lerner RM, Perron M, Pike GB et al (2008) Prenatal exposure to maternal cigarette smoking and the adolescent cerebral cortex. Neuropsychopharmacology 33:1019-1027. https://doi.org/10.1038/sj.npp.1301484

31. Richardson JR, Fitsanakis V, Westerink RHS, Kanthasamy AG (2019) Neurotoxicity of pesticides. Acta Neuropathol. https://doi. org/10.1007/s00401-019-02033-9

32. Pessah IN, Lein PJ, Seegal RF, Sagiv SK (2019) Neurotoxicity of polychlorinated biphenyls and related organohalogens. Acta Neuropathol. https://doi.org/10.1007/s00401-019-01978-1

Publisher's Note Springer Nature remains neutral with regard to jurisdictional claims in published maps and institutional affiliations. 